\title{
Tacrolimus-induced severe cerebral and coronary vasospasm
}

\author{
Laeun Kim, Hye Won Lee
}

Division of Cardiology, Department of Internal Medicine, Pusan National University Hospital, Busan, Korea

Background: Tacrolimus-induced reversible cerebral vasoconstriction syndrome (RCVS) and coronary vasospasm is a rare but not uncommon disease. We describe a patient who presented with tacrolimus induced RCVS and coronary vasospasm together after heart transplantation.

Methods: None.

Results: A 62-year-old female with dilated cardiomyopathy got heart transplantation. Two months later, she complained of a thunderclap headache. Brain computed tomography (CT) showed diffuse cerebral artery constriction with multifocal beaded features at distal intracranial arteries and scanty subarachnoid hemorrhage. We diagnosed it as RCVS induced by tacrolimus. She was at the early period of posttransplant with high risk of rejection, we inevitably maintained a lower level of tacrolimus. By using a vasodilator, the thunderclap headache was disappeared. follow up brain CT showed absorption of hemorrhage and patent cerebral arteries. At 5 months after transplantation, she visited the emergency room with syncope. Shortly after admission, hemodynamic collapsed arrhythmia, and cardiac arrest occurred. We assumed this event as acute rejection and treated her with steroid pulse therapy. Biopsy proved acute rejection G2. She was still at high a risk of rejection, we had to maintain tacrolimus level as high as normal as possible. After the treatment, she complained of chest discomfort with sweating. Telemetry monitoring revealed transient ST-segment elevation when she had the symptoms. We assumed this event was associated with tacrolimus, so we reduced the tacrolimus level. Then, her cardiac symptom was relieved. By using a vasodilator, a coronary vasospasm provocation test was done and revealed severe spasms at the left anterior descending coronary artery. Twenty minutes after the examination, she rapidly collapsed complaining of chest pain. On-site cardiopulmonary resuscitation and extracorporeal membrane oxygenation (ECMO) insertion was made, but 2 days later she died of cardiogenic shock even with ECMO support.

Conclusions: In this case, early detection and withhold tacrolimus is an important strategy. When inevitably maintaining tacrolimus, one should be very cautious in monitoring drug levels and doing the provocation tests. 